\title{
Using Graph Clustering to Locate Sources within a Dense Sensor Array
}

\author{
Nima Riahi (corresponding author) ${ }^{\mathrm{a}}$, Peter Gerstoft ${ }^{\mathrm{a}}$ \\ ${ }^{a}$ Scripps Institution of Oceanography, University of California San Diego, La Jolla, CA 92093
}

\begin{abstract}
We develop a model-free technique to identify weak sources within dense sensor arrays using graph clustering. No knowledge about the propagation medium is needed except that signal strengths decay to insignificant levels within a scale that is shorter than the aperture. We then reinterpret the spatial coherence matrix of a wave field as a matrix whose support is a connectivity matrix of a graph with sensors as vertices. In a dense network, well-separated sources induce clusters in this graph. The geographic spread of these clusters can serve to localize the sources. The support of the covariance matrix is estimated from limited-time data using a hypothesis test with a robust phase-only coherence test statistic combined with a physical distance criterion. The latter criterion ensures graph sparsity and thus prevents clusters from forming by chance. We verify the approach and quantify its reliability on a simulated dataset. The method is then applied to data from a dense 5200 element geophone array that blanketed $7 \mathrm{~km} \times 10 \mathrm{~km}$ of the city of Long Beach (CA). The analysis exposes a helicopter traversing the array and oil production facilities.
\end{abstract}

Keywords: Seismic arrays; Source localization; Graph clustering; Non-parametric estimation

\section{Introduction}

Large and dense sensor arrays are becoming more common as the cost for sensor and communications hardware decreases. Examples of such arrays are the USArray initiative in seismology with 500 stations covering large parts of the continental US [1] or the seismic exploration array with 5200 sensors as presented here. As array sizes increase the occurrence of withinaperture source events that cause coherent signals over only a small fraction of all sensors becomes more common.

This work addresses the problem of localizing such weak sources in a complex and unknown environment. For known or well characterized media this problem has been addressed using frameworks such as matched field processing (MFP), maximum likelihood methods, or migration techniques in, e.g., acoustics $[2,3,4,5]$, seismology $[6,7,8,9,10]$, infrasound acoustics [11, 12], and electromagnetics $[13,14]$. The eigen-structure of the array covariance matrix or its inverse plays an important role in these approaches, in particular for dataadaptive implementations using, e.g., MVDR [15] or MUSIC [16]. A possible solution to locate sources

Email addresses: nriahi@ucsd.edu (Nima Riahi (corresponding author)), gerst of t@ucsd.edu (Peter Gerstoft)

Preprint submitted to Elsevier in dense networks without relying on medium information is the spatiotemporal analysis of signal intensity $[17,18]$. However, using power alone cannot detect sources near or below the noise floor.

We present a model-free analysis approach that can work also for weak signals through the use of coherent averaging. The only assumption made is that source signals enter the noise floor within a distance that is much smaller than the array aperture. That requirement is realistic for large arrays based on wave propagation in moderately attenuating media such as the earth. We follow a graph-based analysis paradigm $[19,20]$ : The sensors are arranged as vertices in a graph with edges between vertices existing if the corresponding sensors share a common coherent signal. Thus the graph connectivity matrix is defined as the support of the array coherence matrix.

For long observation times, we demonstrate that finding weak within-aperture sources is tantamount to identifying connected components in this graph (here referred to as clusters). Such clusters can be found through an eigenvalue decomposition of a matrix (the graph Laplacian) that is derived from the connectivity matrix [21]. In the limited-data case stochastic fluctuations in the coherence can create spurious connections in the graph, thus leading to large connected components, a well known phenomenon studied in random

October 2, 2016 
graph theory. To prevent such spurious clusters from forming we additionally limit the connectivity to the physical neighborhoods of the sensors.

The general applicability of the method comes at the cost of lower precision compared to model-based localization methods: a source is only identified through the sensors on which it has had a significant impact. For the large geophone array (analyzed above $10 \mathrm{~Hz}$ ) we find that the sensor clusters mostly correspond to a small fraction of the array, indicating that high-frequency sources are well determined. We are not aware of any existing techniques that identify such sensor subsets that sense a common source.

The rest of this paper is organized as follows: Section 2 defines the coherence hypothesis test, introduces some graph concepts, and describes how a graph is constructed from the array coherence matrix. In Section 3 a proof is given that within-array sources induce clusters in that graph in the asymptotic case assuming sufficient spatial signal decay. We verify and test the reliability of finding sources using these clusters on simulated data in Section 4. This is followed in Section 5 by an application to real data from a 5200 sensor geophone array that covered $70 \mathrm{~km}^{2}$ of the city of Long Beach (CA) with a sensor spacing of about $100 \mathrm{~m}$.

\section{Coherence matrix defines a graph}

Consider a large aperture array with $N$ sensors distributed densely over spatial locations $\left\{\mathbf{r}_{i}\right\}_{i=1, \ldots, N}$. The arrays we consider are quasi-uniform, but different configurations are applicable. This section first describes a hypothesis test to find the support of the coherence matrix of these sensors and then describes how to use this support to construct a graph and find its clusters. Finally, we investigate the clustering behavior in a sourcefree network.

\subsection{Coherence hypothesis test}

\subsubsection{Robust coherence}

We compare the behavior of two definitions of magnitude of coherence for uncorrelated and heteroscedastic signals, i.e. stochastic signals with time-varying variance. Consider a zero-mean signal $u_{j}(t)$ observed at location $\mathbf{r}_{j}$ and captured by a window of $Q$ samples at intervals $\Delta t$. Its discrete Fourier transform over a period $T_{W}=Q \Delta t$ during the $m$-th window (snapshot) is defined as:

$$
x_{j}(m, f)=\sum_{q=0}^{Q-1} w_{q} u_{j}(m Q \Delta t+q \Delta t) e^{-l 2 \pi(q \Delta t) f},
$$

where the frequencies are discretized $f=\frac{k}{T_{W}}, k=$ $0, \ldots, Q / 2$ and the weights $w_{q}$ control spectral leakage [22]. All the considerations in this article are made in the Fourier domain.

Let $x_{i}(m)$ and $x_{j}(m)$ be a sequence of snapshots of two such signals in the frequency domain, $m=1, \ldots, M$. The sample covariance is defined as

$$
\hat{\Gamma}_{i j}=\frac{1}{M} \sum_{m=0}^{M-1} x_{i}(m) x_{j}^{*}(m)
$$

with the actual covariance reached for infinite snapshots:

$$
\hat{\Gamma}_{i j} \stackrel{M \rightarrow \infty}{\longrightarrow} \Gamma_{i j}
$$

The sample covariance estimate will be affected by the variances in $x_{i}$ and $x_{j}$ that are unrelated to any physical relation between the two sensors. A customary attempt to reduce the impact of such variations is to compute the coherence as a normalized covariance. We compare two definitions of magnitude of coherence. A full-sample normalized coherence:

$$
\hat{C}_{i j}^{c}=\left|\frac{\frac{1}{M} \sum_{m=0}^{M-1} x_{i}(m) x_{j}^{*}(m)}{\left(\frac{1}{M} \sum_{m=0}^{M-1}\left|x_{i}(m)\right|^{2}\right)^{1 / 2}\left(\frac{1}{M} \sum_{m=0}^{M-1}\left|x_{j}(m)\right|^{2}\right)^{1 / 2}}\right|,
$$

and a version relying only on phase-information:

$$
\hat{C}_{i j}=\left|\frac{1}{M} \sum_{m=0}^{M-1} \frac{x_{i}(m)}{\left|x_{i}(m)\right|} \frac{x_{j}^{*}(m)}{\left|x_{j}(m)\right|}\right|,
$$

The coherence (5) only relies on phase information and is invariant against heteroscedasticity, i.e. signals with time-varying intensity [23]. This is important because such signals are common in seismic and acoustic timeseries. To illustrate the effect of heteroscedasticity we consider three scenarios for $x_{i}$ and $x_{j}$ as shown in Table 1, where $x_{i}$ and $x_{j}$ are uncorrelated and each i.i.d. complex Gaussian with a variance that depends on the snapshot index. Figure 1A shows the simulated pdf of $\hat{C}_{i j}^{c}$ for the three scenarios (based on $10^{6}$ realizations). The pdf of the sample coherence $\hat{C}_{i j}^{c}$ substantially deviates from the stationary case for the two non-stationary scenarios considered. This instability with respect to heteroscedasticity makes $\hat{C}_{i j}^{c}$ a poor choice for a hypothesis test against independence. Figure 1B shows the pdf of $\hat{C}_{i j}$ for the same scenarios as before and demonstrates how the distribution of this statistic is invariant for the considered heteroscedasticity scenarios. 
Table 1: Three scenarios considered for $M=19$ snapshots of the noise processes $x_{i}$ and $x_{j}$ (see Figure 1). $x_{i}(q)$ referes to the $q$-th snapshot of process $x_{i}$

\begin{tabular}{|l|c|c|}
\hline \multicolumn{1}{|c|}{ Scenario } & Variance $x_{i}$ & Variance $x_{j}$ \\
\hline \hline \multicolumn{1}{|c|}{ Stationary } & $\sigma_{i}^{2}(1 \ldots 19)=1$ & $\sigma_{j}^{2}(1 \ldots 19)=1$ \\
\hline Heteroscedastic & $\sigma_{i}^{2}(1 \ldots 5)=10$ & $\sigma_{j}^{2}(1 \ldots 5)=10$ \\
& $\sigma_{i}^{2}(6 \ldots 19)=1$ & $\sigma_{j}^{2}(6 \ldots 19)=1$ \\
\hline Heteroscedastic & $\sigma_{i}^{2}(1 \ldots 5)=10$ & $\sigma_{j}^{2}(1 \ldots 14)=1$ \\
2 & $\sigma_{i}^{2}(6 \ldots 19)=1$ & $\sigma_{j}^{2}(15 \ldots 19)=10$ \\
\hline
\end{tabular}

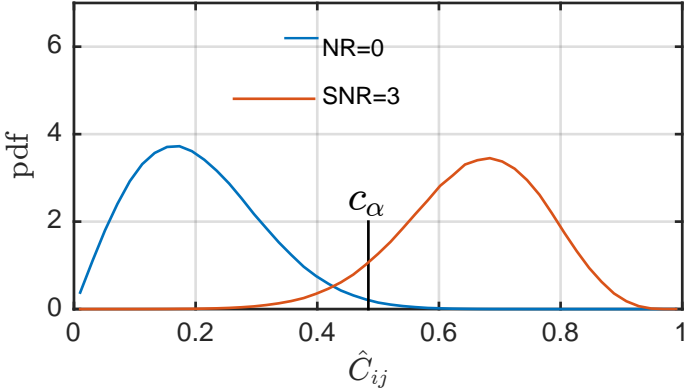

Figure 2: The PDF (blue) of the magnitude sample coherence $\hat{C}_{i j}(5)$ for uncorrelated noise, $\Gamma_{i j}=0$, for $M=19$. The decision threshold $c_{\alpha}=0.484$ is exceeded with probability $\alpha=0.01$. The PDF (orange) of $\hat{C}_{i j}$ for the case of $\Gamma_{i j} \neq 0$ with a common signal present in the noise of the two recordings $(\mathrm{SNR}=3)$.

\subsubsection{Hypothesis test}

We describe a hypothesis test with robust teststatistics to establish the support of the array coherence matrix $\Gamma_{i j}$ (3). We test for the two alternative hypotheses:

$$
\begin{aligned}
& H_{0}: \Gamma_{i j}=0, \\
& H_{1}: \Gamma_{i j} \neq 0,
\end{aligned}
$$

i.e. the signals observed at locations $\mathbf{r}_{i}$ and $\mathbf{r}_{j}$ are uncorrelated $\left(H_{0}\right)$ or correlated $\left(H_{1}\right)$. We use the magnitude of the robust sample coherence $\hat{C}_{i j}$ (5) to test the hypothesis. If $H_{0}$ is true, then $\hat{C}_{i j}$ will be distributed according to a pre-computable PDF (Figure 2 shows the PDF for $M=19$ derived by simulation). The hypothesis $H_{0}$ is accepted if $\hat{C}_{i j} \leq c_{\alpha}$ and rejected otherwise. The threshold coherence magnitude $c_{\alpha}$ is set such that the probability of falsely rejecting the hypothesis is $\alpha$, formally $c_{\alpha}=\operatorname{cdf}^{-1}(1-\alpha)$, where $\operatorname{cdf}^{-1}(\cdot)$ is the inverse of the cumulative distribution function of $\hat{C}_{i j}$ estimated by simulation (blue plot in Figure 2).

Figure 3 shows how $c_{\alpha}$ decreases monotonically with increasing number of snapshots $M$. For $M=19$ we have $c_{\alpha}=0.484$. Note that if the travel time difference between two sensors exceeds $T_{W}$ then the contribution of that source to the coherence is zero. While a large $M$ is preferred for statistical reasons, due to the nonstationary sources $M$ cannot be too large.

To provide an idea about the likelihood of falsely accepting the null-hypothesis (e.g. a misdetection) Figure 2 also shows the simulated PDF of $\hat{C}_{i j}$ for the case where there is a signal present: $x_{i}=s+n_{i}$ and $x_{j}=s+n_{j}$ where $P_{s}=\operatorname{var}(s)$ and $P_{n}=\operatorname{var}\left(n_{i}\right)=\operatorname{var}\left(n_{j}\right)$ and SNR $=P_{s} / P_{n}=3$, i.e. $H_{1}$ is true. The false acceptance probability for $H_{1}$ is 0.0768 (area under orange curve below $c_{\alpha}$ ). 


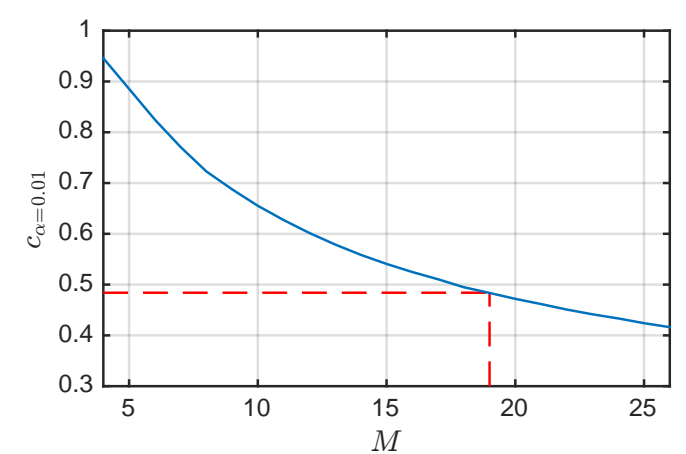

Figure 3: The decision threshold $c_{\alpha}(\alpha=0.01)$ as a function of number of snapshots $M$. For $M=19$ we have $c_{\alpha}=0.484$.

\subsection{Graph preliminaries}

An undirected (symmetric) and unweighted graph $G$ consists of $N$ vertices $\left\{v_{i}\right\}_{i=1 \ldots N}$ and edges $\left\{e_{i j}\right\}_{j \geq i} \in\{0,1\}$ where $e_{i j}=1$ means that $v_{i}$ and $v_{j}$ are connected. The edges define a binary and symmetric connectivity matrix $\mathbf{E}$ with $E_{i j}=e_{i j}$. The number of edges connecting to vertex $v_{i}$ is its degree $d_{i}$. The mean vertex degree $\gamma$ of a graph is the average over the vertex degrees of all its vertices. If $\gamma$ approaches a constant as $N$ increases the graph is sparse [24].

A connected component $U \subset G$ is a subset of vertices and edges in $G$ for which every pair of vertices $v, v^{\prime} \in U$ is connected directly or indirectly through a sequence of edges in $U$ (see Figure 4). Finding connected components is a basic task in graph analysis [25] and is an example of spectral clustering for non-overlapping clusters. In this context the connected components are found using the eigen-vectors of the graph Laplacian, which is derived from the connectivity matrix $\mathbf{E}$ [21]. We assume now that there are $K$ fully connected components $U^{k}$ in $G$, i.e. each vertex in $U^{k}$ connects to all other vertices in $U^{k}$. Let $\mathbf{u}^{k}=\left[u_{1}^{k}, \ldots, u_{N}^{k}\right]^{T}$ be the vertex indicator vector of $U^{k}\left(u_{i}^{k}=1\right.$ if $v_{i}^{k} \in U^{k}$ and 0 otherwise). The connectivity matrix of $G$ is then (see Appendix A):

$$
\mathbf{E}=\sum_{k} \mathbf{u}_{k} \mathbf{u}_{k}^{T},
$$

where $k$ indexes the set of connected components of $G$. Note that any graph where the same vertices are connected in groups has the same or a smaller support than E.

Finally, consider the random, unweighted graph $G_{0}(N, p)$ with $N$ vertices where all pairs of vertices have the same probability $p$ of being connected. The mean vertex degree in $G_{0}(N, p)$ is therefore $\gamma=(N-1) p$ because every vertex can connect with all $N-1$ other ver-

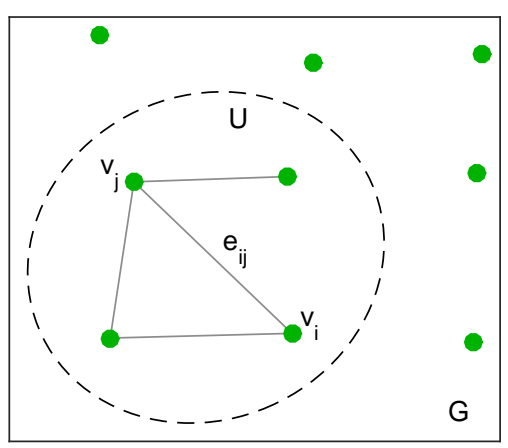

Figure 4: A sample graph $G$ with $N=9$ vertices (circles) and four edges (lines). The connected component $U$ consists of the vertices and edges within the dashed area. Vertex $v_{j}$ has degree $d_{j}=3$, and $v_{i}$ degree $d_{j}=2$. The mean vertex degree $\gamma=(5 * 0+1+2 * 2+3) / 9=8 / 9$.

tices with equal probability. A large fraction of vertices in a random graph tend to be connected when $\gamma>1$ with about $90 \%$ being connected when $\gamma>2.5$ [24]. Such a high connectivity, e.g. 90\%, will thus occur above a threshold probability of

$$
p_{0}=2.5 /(N-1)
$$

i.e. for an edge probability above $p_{0}$ most vertices will be connected. This upper limit decreases as the graph grows larger. For a graph with $N=300$ vertices this threshold is already below 0.01 . As we'll see later, preventing large clusters from forming by chance is important because the clusters will be used to detect sources within a sensor network.

\subsection{Constructing an array graph}

Armed with the hypothesis test in Section 2.1.2 we construct a coherence graph $G_{0}$ with the following connectivity matrix:

$$
E_{i j}^{0}= \begin{cases}1 & \text { if } \hat{C}_{i j}>c_{\alpha} \\ 0 & \text { otherwise }\end{cases}
$$

i.e. two vertices are connected if the corresponding signals exhibit significant coherence. This straight-forward construction of an array graph, however, is insufficient because of the statistical fluctuations of the hypothesis test. Even if the array is sensing $N$ uncorrelated noise signals the probability of observing $\hat{C}_{i j}>c_{\alpha}$ is $\alpha$ for all receiver pairs. This means that $G_{0}$ is a random graph $G_{0}(N, \alpha)$. As seen in (8) a graph with, say, 300 sensors will likely have a giant connected component if $\alpha>2.5 /(300-1)=0.008$. For graphs constructed with a less conservative threshold any attempt to find smaller 


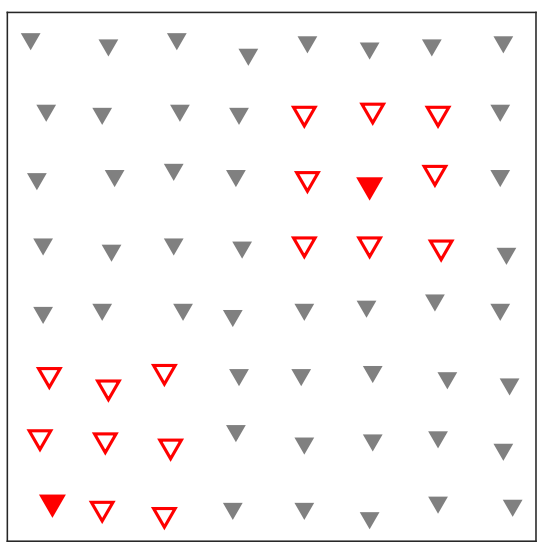

Figure 5: Connections between a sensor are only allowed to its nearest neighbors. The eight nearest neighbors (red open triangles) are shown for two example sensor locations (red triangles).

connected components that are not due to chance is thus futile.

We modify (9) to define a localized coherence graph $G\left(c_{\alpha}\right)$ with connectivity matrix:

$$
E_{i j}= \begin{cases}1 & \text { if } \hat{C}_{i j}>c_{\alpha} \text { and } i \in \mathrm{N}(j) \\ 0 & \text { otherwise. }\end{cases}
$$

where $\mathrm{N}(j)$ is the index set of the nearest neighbors of sensor $j$. For a regular lattice, the nearest neighbors are here limited to eight sensors as shown in Figure 5. Besides being coherent any two connected sensors are thus also required to be spatial neighbors.

Enforcing spatially short connections limits the number of neighbors any vertex can connect to in a way that is independent of the global graph size and the graph remains sparse for large arrays. The criterion (10) thus reduces the chance of forming clusters by chance, even for values of $\alpha$ that are above the threshold suggested by (8). Sensor clusters can still have a spatial extent beyond that given by the nearest neighbors as long as the vertices in the cluster are contiguous in space.

To characterize the spatial extent of each connected component $U^{k}$ a two-dimensional Gaussian probability density function is estimated from the sensor locations of the vertices of $U^{k}$ with mean and covariance, respectively:

$$
\begin{aligned}
\mathbf{m}_{k} & =\frac{1}{\left|U^{k}\right|} \sum_{i \in U^{k}} \mathbf{r}_{i} \\
\mathbf{\Sigma}_{k} & =\frac{1}{\left|U^{k}\right|} \sum_{i \in U^{k}}\left(\mathbf{r}_{i}-\mathbf{m}_{k}\right)\left(\mathbf{r}_{i}-\mathbf{m}_{k}\right)^{T},
\end{aligned}
$$

where $\left|U^{k}\right|$ is the number of vertices in $U^{k}$. The source area is the region where the point source is likely located and is here defined as the ellipse that contains a probability mass $p$ of the Gaussian defined in (11):

$$
\Omega_{k}(p)=\left\{\mathbf{r} \mid\left(\mathbf{r}-\mathbf{m}_{k}\right)^{T} \boldsymbol{\Sigma}_{k}^{-1}\left(\mathbf{r}-\mathbf{m}_{k}\right)<\chi_{I}^{2}(p)\right\},
$$

where $\chi_{I}^{2}$ is the cumulative inverse $\chi^{2}$-distribution with two degrees of freedom (because the Gaussian is 2D). An effective source diameter $d_{\text {eff }}$ of a disk with the same area $A\left(\Omega_{k}\right)$ as the source ellipse $\Omega_{k}$ is defined as:

$$
d_{\mathrm{eff}}=2 \sqrt{A\left(\Omega_{k}\right) / \pi} .
$$

For a source within the array aperture $\Omega$ is the geographic area within which the source is estimated to be.

As will be seen in section 4 this definition of source area works well for the simulation considered, but source directionality, physical obstacles or attenuation heterogeneities in the propagation medium can cause $\Omega$ to be not centered around a source. Sensor geometry such as array gaps and boundaries will also cause a cluster to move away from its source. In those special cases the identified clusters can, however, still serve to select a data subset for follow-up analysis with other array processing methods since by definition its sensors contain significant signal levels from a common source. E.g. conventional beamforming using just the data from the vertices in $U^{k}$.

\subsection{Size of connected components in noise-only array}

As discussed before the distance constraint in (10) prevents connected components to form by chance even in large arrays. We demonstrate this for a rectangular array of size $2.8 \times 2.8 \mathrm{~km}$ with sensor spacing of $\sim 100 \mathrm{~m}$ and 841 sensors (gray triangles in Figure 6). We consider $M=19$ snapshots of random complex Fourier coefficients, as this is the number of snapshots also used later in the analysis sections 4 and 5. For each vertex, the $M=19$ snapshots are simulated as random complex numbers drawn from the complex Gaussian distribution and the sample coherence matrix $\hat{C}_{i j}(5)$ is formed. The corresponding array graph $G\left(c_{\alpha=0.01}\right)$ is constructed and the number of vertices and edges of the connected component with the most vertices is stored. The procedure is repeated 200'000 times. Figure 7 gives the fraction of simulations where the vertices exceeded a given number. For the later analysis for noise affected array data we are interested in a minimum size criterion that rejects as many random components as possible while not being overly conservative. Note that large values for these criteria will increase the cluster size and hence the resolution with which sources can be localized. 


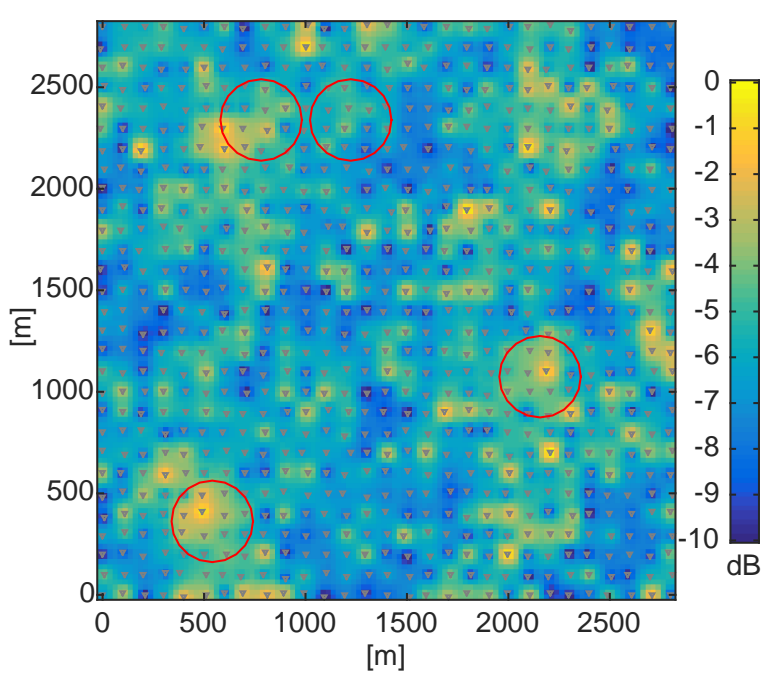

Figure 6: Simulation layout showing 841 sensors in a regular configuration (gray triangles). The four sources are located at the center of the red circles. An interpolated map of signal power across the array averaged over $M=19$ snapshots is shown in the background. The log-normally distributed noise power prevents the sources from appearing as unique peaks.

Requiring a minimum of seven vertices seems a safe criterion because none of the simulations gave rise to such large components. But this would unduly limit the size of the smallest resolvable cluster. The smallest tolerable minimum cluster size is four vertices, which occurred in $10.8 \%$ of simulations. This fraction seems high at first, but upon closer scrutiny we find that of the clusters in these simulations only $9.3 \%$ contained four or more edges, with all the others having three edges. We therefore settle on a minimum criterion of four vertices and four edges, in which case only $10.8 \% \times 9.3 \%=1.0 \%$ of random simulations would pass the criterion.

\section{Sources induce graph clusters}

The relation between sources within an array and the clusters of a graph constructed from the array data has been presumed so far. Combining signal features for clustering purposes to analyze sources was used implicitly in a heuristic approach in [26] for the difficult case of an ad-hoc and dynamic sensor network with communication constraints. In this section we make the relation between sources and network clusters explicit for the asymptotic case of infinite observation time without communications constraints but under the assumption that source-to-receiver coherence is insignificant after some physical distance.

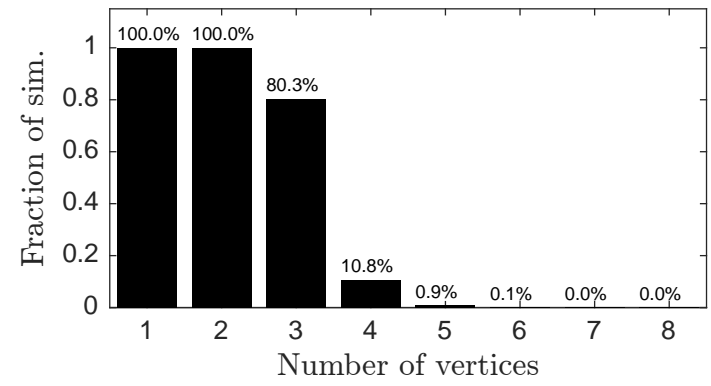

Figure 7: Histogram over the largest cluster size in 200,000 random realizations of a localized coherence graph $G\left(c_{\alpha}\right)$. In most random graphs the largest cluster has three vertices. Of all simulations $11 \%$ contained at least one cluster that had four or more vertices, but only $0.08 \%$ had four or more vertices as well as four or more edges.

Consider again a large aperture array with $N$ sensors distributed densely over spatial locations $\left\{\mathbf{r}_{i}\right\}_{i=1, \ldots, N}$. It is assumed that there are weak sources within the aperture that produce signals that propagate through space. For a given frequency the channel between any such source location $\boldsymbol{\rho}$ and sensor location $\mathbf{r}_{i}$ is characterized by a Green's function, $g\left(\mathbf{r}_{i}, \boldsymbol{\rho}\right)$. Let the vector $\mathbf{g}(\boldsymbol{\rho})=\left[g\left(\mathbf{r}_{1}, \boldsymbol{\rho}\right), \ldots, g\left(\mathbf{r}_{N}, \boldsymbol{\rho}\right)\right]^{T} \in \mathbb{C}^{N}$ be the frequency domain response of the array to a source at location $\boldsymbol{\rho}$. Consider then $\boldsymbol{\rho}_{k}$ to be the location of $K$ sources $\left\{\boldsymbol{\rho}_{k}\right\}_{k=1, \ldots, K}$ with an associated response $\mathbf{g}\left(\boldsymbol{\rho}_{k}\right) \equiv \mathbf{g}_{k}$ and source signals $s_{k}$. The measured signal at the $N$ array sensors is thus modeled as:

$$
\mathbf{x}=\sum_{k=1}^{K} s_{k} \mathbf{g}_{k}+\mathbf{n} .
$$

where $\mathbf{n}=\left[n_{1} \ldots n_{N}\right]^{T} \in \mathbb{C}^{N}$ is a multivariate i.i.d. noise process. From (14) the covariance matrix is:

$$
\begin{aligned}
\boldsymbol{\Gamma} & =\left\langle\mathbf{x} \mathbf{x}^{H}\right\rangle=\left\langle\sum_{k, l=1}^{K} s_{k} s_{l}^{*} \mathbf{g}_{k} \mathbf{g}_{l}^{H}\right\rangle+\left\langle\mathbf{n n}^{H}\right\rangle \\
& =\sum_{k, l=1}^{K}\left\langle s_{k} s_{l}^{*}\right\rangle \mathbf{g}_{k} \mathbf{g}_{l}^{H}+\mathbf{D} \\
& =\sum_{k=1}^{K}\left\langle\left|s_{k}\right|^{2}\right\rangle \mathbf{g}_{k} \mathbf{g}_{k}^{H}+\mathbf{D},
\end{aligned}
$$

where $(\cdot)^{H}$ is the complex conjugate transpose and $\langle\cdot\rangle$ is the expectation over infinite time. Here we exploit the mutual independence between the source and noise processes $\left\langle s_{i} n_{j}^{*}\right\rangle=0,\left\langle n_{i} n_{j}^{*}\right\rangle=\left\langle s_{i} s_{j}^{*}\right\rangle=\delta_{i j}, \forall i, j$, and D is a diagonal matrix with $D_{i i}$ the noise variance of sensor $i$.

We assume that there is a distance $\delta$ smaller than the array dimensions such that $g\left(\mathbf{r}_{i}, \boldsymbol{\rho}\right)$ is small if $\left\|\boldsymbol{\rho}-\mathbf{r}_{i}\right\|_{2}>$ 
$\delta$, i.e. signals cannot be detected beyond $\delta$. This is used to form connected graph for each source. If all sources are separated by at least $2 \delta,\left\|\rho_{k}-\rho_{l}\right\|_{2}>2 \delta \quad \forall k \neq l$, then the corresponding support sets of the sources do not overlap.

Let $\mathcal{I}(\mathbf{v})$ be the support indicator function of a vector or matrix $\mathbf{v}$. The lack of overlap of the $\mathbf{g}_{k}$ and the support-indicator function properties (see Appendix B) allow us to write the support of the sum in (15) as

$$
\begin{aligned}
\mathcal{I}(\boldsymbol{\Gamma}) & =\mathcal{I}\left(\sum_{k=1}^{K}\left|s_{k}\right|^{2} \mathbf{g}_{k} \mathbf{g}_{k}^{H}\right)=\sum_{k=1}^{K} \mathcal{I}\left(\mathbf{g}_{k} \mathbf{g}_{k}^{H}\right) \\
& =\sum_{k=1}^{K} \mathcal{I}\left(\mathbf{g}_{k}\right) \mathcal{I}\left(\mathbf{g}_{k}\right)^{T}
\end{aligned}
$$

We now define $E_{i j}=I(\boldsymbol{\Gamma})$ as the connectivity matrix of a graph $G$ with $N$ vertices (sensors), i.e. there is an edge between vertices $i$ and $j$ if $E_{i j}=\mathcal{I}(\mathbf{C})_{i j}=1$. As shown in Appendix A such a graph $G$ will have exactly $K$ connected components, i.e. $K$ non-overlapping subsets $S^{k} \in G$ whose vertices are indicated by $\mathcal{I}\left(\mathbf{g}_{k}\right)$. The connected components thus correspond to the sensor clusters that sensed the $K$ sources.

Finding connected components is a standard task in graph analysis [25]. In the coming sections 4 and 5 we use an approach from spectral clustering [21] which uses the eigendecomposition of the graph Laplacian, which is derived from the connectivity matrix as

$$
\mathbf{L}=\mathbf{K}-\mathbf{E},
$$

with $\mathbf{K}$ a diagonal matrix with $\mathbf{K}_{i i}=\sum_{j=1}^{N} E_{i j}$. Let the eigen-decomposition of $\mathbf{L}$ be

$$
\mathbf{L}=\mathbf{U S} \mathbf{U}^{T} .
$$

Following [21, Proposition 2] there will be exactly $K$ eigenvectors with eigenvalue 0 and the column vectors in $\mathbf{U}$ corresponding to those eigenvectors will each indicate one of the $K$ connected components through its non-zero entries.

\section{Verification on Simulated Data}

Consider a homogeneous half-space with medium propagation velocity $c=340 \mathrm{~m} / \mathrm{s}$ and a square array on its surface with aperture $2.8 \mathrm{~km} \times 2.8 \mathrm{~km}$. The sensors are placed in a regular lattice configuration spaced $100 \mathrm{~m}$ apart, i.e. $N=29 \times 29=841$ sensors at positions $\mathbf{r}_{1}, \ldots, \mathbf{r}_{N}$ (Figure 6). Four point-sources are simulated at random locations $\boldsymbol{\rho}_{k}$ (red circles) with uncorrelated complex Gaussian source signals $s_{k}(t) \sim \mathcal{C N}\left(0, \sigma_{k}^{2}\right)$, $k=1, \ldots, 4$. Furthermore, each sensor is affected by some additive noise $n_{i}(t) \sim \mathcal{C N}\left(0, \sigma_{i}^{2}\right), i=1, \ldots, N$. The noise intensities $\sigma_{i}^{2}$ are spatially uncorrelated and follow a log-normal distribution, an empirical phenomenon observed in real data [18]. The time-domain observation at location $\mathbf{r}_{i}$ is:

$$
u_{i}(t)=\sum_{k=1}^{4} \frac{s_{k}\left(t-\frac{\left|\mathbf{r}_{i}-\boldsymbol{\rho}_{k}\right|}{c}\right)}{\left\|\mathbf{r}_{i}-\boldsymbol{\rho}_{k}\right\|_{2}}+n_{i}(t) .
$$

The signal-to-noise ratio is defined at a distance of $100 \mathrm{~m}$ to the source as SNR $=\frac{R}{100^{2} \bar{P}}$, where $\bar{P}$ is the average noise power over all sensors. The simulated data is transformed into the Fourier domain, $x_{i}(m)$, using (1).

Figure 6 shows an interpolated map of measured seismic power at $20 \mathrm{~Hz}$ over the array at $S N R=1$. The simple localization of the sources by means of finding peaks in this map is prevented by the log-normally distributed noise power: it leads to many high power locations that are purely a function of local noise.

Given $x_{i}(m)$ we use (5) to obtain a sample coherence matrix $\hat{C}_{i j}$ for $M=19$ snapshots. From this a graph $G\left(c_{\alpha=0.01}\right)$ is constructed using (10) and the connected components with more than four vertices and four edges (see Section 2.4) are identified as potential source indicators. The location and size of the estimated source area is calculated from (11) and (12) as $\mathbf{m}$ and $\Omega(p=$ $0.5)$.

To demonstrate how the connected graph is constructed we focus on the SNR $=10$ and $\alpha=0.01$ case in Figure $8 \mathrm{a}$. Computing the coherence for a sequence of sensors from South to North (yellow asterisks) and applying the nearest neighbor localization introduced in eq. (10) gives a banded localized coherence matrix $\hat{C}_{i j}$, Figure $8 \mathrm{~b}$, where the sensors are numbered column-vice from southwest to northeast. The center diagonal corresponds to the sensor itself and is set zero, the first offdiagonal corresponds to the sensor above and below in Figure $8 \mathrm{a}$. Since each column has 29 sensors, the 3 subdiagonals \pm 29 entries away corresponds to the 3 left and right sensors. Testing if $\hat{C}_{i j}>\alpha$ defines the graph connectivity matrix $E_{i j}$ according to eq. (10), see Figure $8 \mathrm{c}$. Each element $E_{i j}=1$ represents a connection between sensors $i$ and $j$. The black asterisks in Figure 8a show all connections other than those to the yellow asterisks.

In Figure 9 we perform a simulation for four fixed sources over a wide range of SNR and $\alpha$ values. It is apparent how higher SNR and higher $\alpha$ tend to increase the size and the number of detected clusters. Note that a cluster indicates all sensors that are sensing the signal with sufficient strength, explaining why at high SNR 

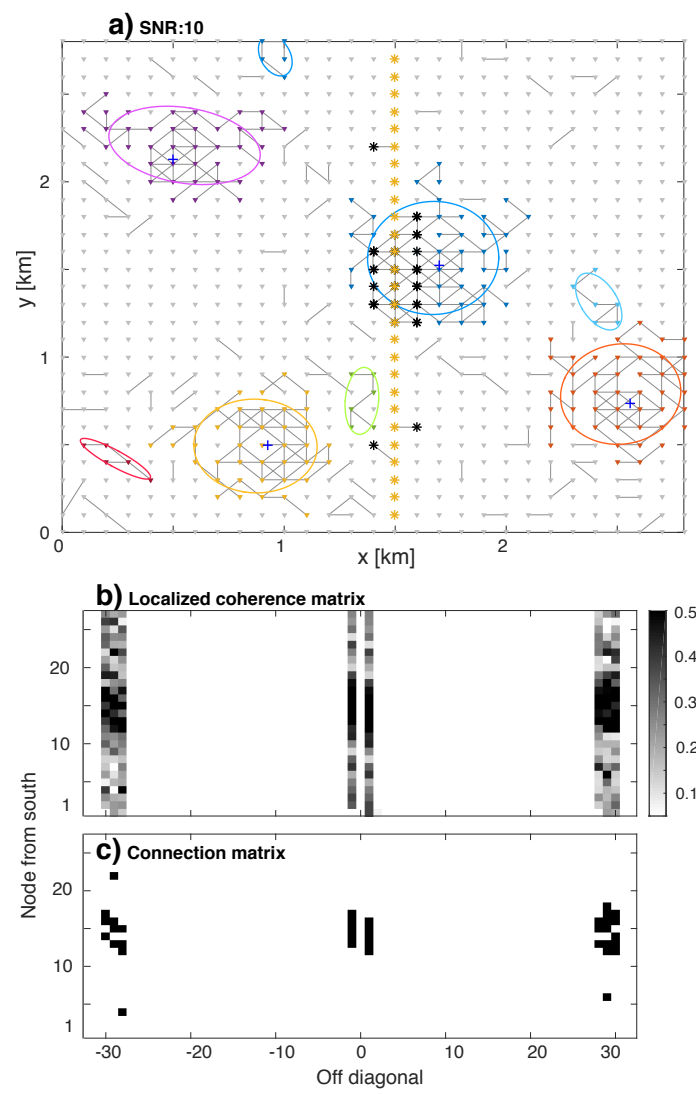

Figure 8: (a) The sensor graph constructed from the simulated data for $\mathrm{SNR}=10$ and $\alpha=0.01$. The plot shows the array (gray triangles) with the graph edges (gray lines). Connected components with more than four vertices and four edges are highlighted with an ellipse $\Omega(0.50)$ following eqs. (11)-(12). In addition, for a North-South line of sensors (yellow asterisks) all nodes connecting to it are shown (black asterisks). (b) Non-zero entries of the localized coherence matrix $\hat{C}_{i j}$ for the column of nodes in (a) (yellow asterisks). Only the 32 nearest elements to the diagonal element are shown. (c) The connectivity matrix $E_{i j}$ corresponding to $(b)$. a larger cluster size is obtained. As can be seen with the blue clusters in Figure 9 for high SNR and $\alpha$ this can limit resolution as the clusters of two or even three distinct sources coalesce into one, occasionally losing some sources in the process (e.g. bottom right panel). High SNR and low $\alpha$ or low SNR and high $\alpha$ tend to create spurious clusters but mostly yield cluster ellipses that capture the true source locations. The clusters formed at low SNR are likely due to random fluctuations as discussed for a random graph in section 2.4. From this visualization we can qualitatively infer that the method should be able to detect sources well at intermediate SNR.

As discussed above the clusters can coalescence for high SNR and $\alpha$. However, source clusters tend to be more highly connected near the location of their coherence-inducing source as opposed to periphery, especially if the number of nearest neighbors in the localization step, eq. (10), is increased. In that case there is evidence that even coalesced clusters can be disentangled using graph analysis techniques of community detection [27, 28, 29].

The performance over 500 Monte Carlo simulations is quantified for SNR from $0.5-5$ and four $\alpha$ values in Table 2. In each simulation there are four actives sources placed at random locations within the array, yielding a total of 2000 sources. We compute the fraction of these sources that are enclosed by the $\Omega(0.5)$ ellipse of any of the detected clusters (source detection rate). To capture how specific these detections are the area covered by the largest ellipse that occurred in the simulation is also stored. Note that the source detection rate can start to deteriorate when moving to high SNR and $\alpha$. This is likely attributable to the coalescence of several ellipses into one larger and more centered ellipse which can miss some sources as discussed earlier in Figure 9. Once a cluster is identified further analysis, e.g. using matched-field processing on the largest clusters, could identify the source location more precisely or find several sources within the cluster.

\section{Long Beach (CA) Geophone Array}

To demonstrate the above technique we apply it on a geophone array that was deployed over an area of $7 \times 10 \mathrm{~km}$ in Long Beach (California, US) as part of an industrial seismic survey $[18,30,31,32,33,34]$. The dataset is purely observational and there is little ground truth available for it. For this reason, the following exposition will serve as a rough qualitative assessment of the technique. 


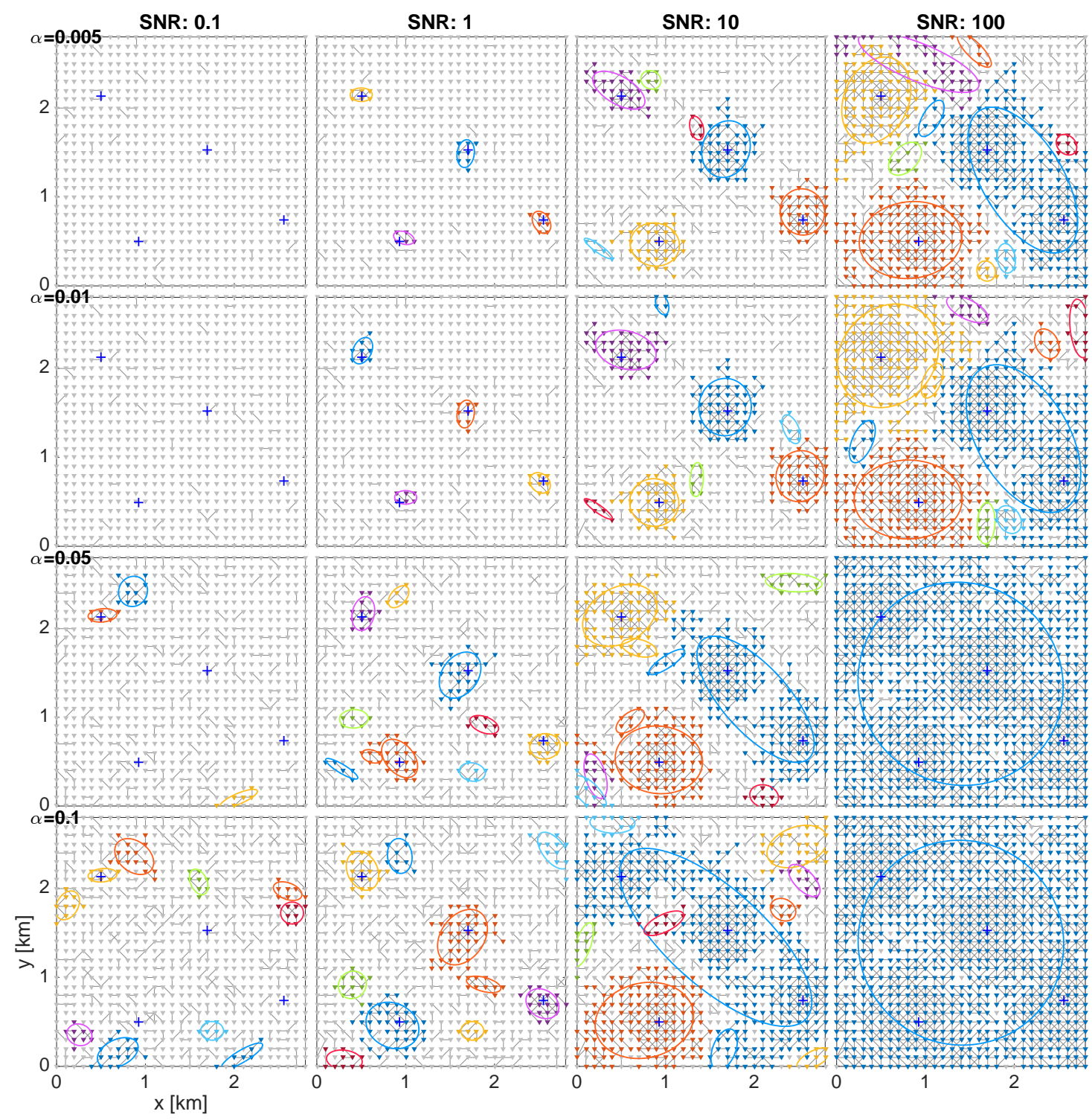

Figure 9: The sensor graph constructed from the simulated data for (first column) $\mathrm{SNR}=0.1$, (second column) $\mathrm{SNR}=1$, (third column) $\mathrm{SNR}=10$, and (fourth column) SNR $=100$ and (first row) $\alpha=0.005$, (second row) $\alpha=0.01$, (third row) $\alpha=0.05$, and (fourth row) $\alpha=0.1$. Each plot shows the array (gray triangles) with the gray lines showing graph edges. Connected components with more than four vertices and four edges are highlighted with an ellipse $\Omega(0.50)$ following eqs. (11)-(12). 
Table 2: Fraction of sources enclosed by a cluster ellipse (source detection rate) based 500 Monte Carlo simulations (above) and average area spanned by the largest ellipse relative to the entire array area (bottom).
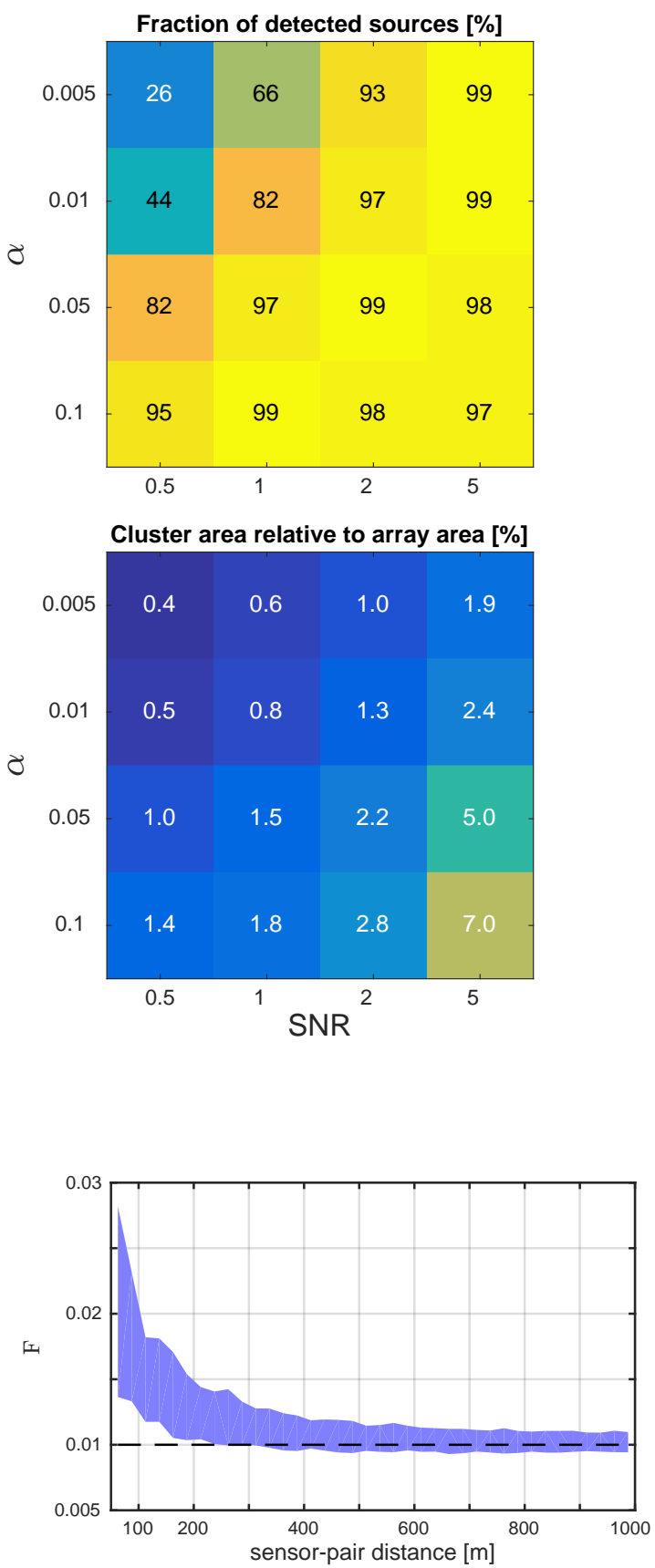

Figure 10: Fraction $F$ of sensor pairs for which the null hypothesis is rejected at $\alpha=0.01$ versus sensor separation $d_{i j}=\left\|\mathbf{r}_{i}-\mathbf{r}_{j}\right\|_{2}$ (at $20 \mathrm{~Hz}$ ). The data was taken from a 12 hour period on March 10th 08.00-20.00h. The shaded area indicates the 10-90 percentiles.
The array consisted of more than 5200 geophones (OYO CT32D vertical velocity sensors with $10 \mathrm{~Hz}$ corner frequency) sampling at a period of $\Delta t=4 \mathrm{~ms}$ (array configuration shown in Figure 11A). For the most part the array had a quasi-regular layout with a relatively even spatial sampling.

The ground velocity data stream of each geophone is transformed following (1) into a sequence of Fourier coefficients $x_{i}(m, f)$ using $Q=256$ samples $\left(T_{W}=1.02 \mathrm{~s}\right)$ and a Hanning window $w_{j}$ with time windows overlapping by $T_{W} / 2$. The coherence matrix $\hat{\mathbf{C}}_{i j}(5)$ is computed for 41 frequency bins from $9.8-48.8 \mathrm{~Hz}$ using $M=19$ snapshots $\left(T_{W} / 2 \times(M+1)=10.2 \mathrm{~s}\right)$. A matrix with about $5200^{2} \approx 27 \cdot 10^{6}$ entries is therefore computed for every frequency bin and time period. In a 24 hour analysis period there are about 9400 time windows.

First, an analysis of the spatial scale of coherence is performed. The receiver-pairs are grouped by spatial distance $d_{i j}=\left\|\mathbf{r}_{i}-\mathbf{r}_{j}\right\|_{2}$ into bins of width $25 \mathrm{~m}$. The average spacing is $110 \mathrm{~m}$ For distance bin $k$, (i.e., $0<$ $\left.d_{i j}-k * 25<25\right)$ the fraction of pairs that reject the null hypothesis is computed:

$$
F_{k}=\frac{\#\left\{i, j \text { in bin } k \mid \hat{C}_{i j}>c_{\alpha}\right\}}{\#\{i, j \text { in bin } k\}},
$$

where \#\{\} is the cardinality of the set. A high value of $F$ thus indicates that the signals measured by receiverpairs within a distance bin tend to be coherent. The process is repeated for all time segments from March 10th 08.00-20.00h (all time indications are local time). Figure 10 shows $F$ versus receiver-pair distance for frequency $20 \mathrm{~Hz}$. Its value, and thus coherence, is clearly elevated for shorter distances but reaches the level $\alpha=0.01$ at distance above about $400 \mathrm{~m}$. This indicates that local coherent phenomena occur regularly in the data. As indicated in Section 2.1.2 the coherence must drop to zero for travel time differences above the analysis window length $T_{W}=1.02 \mathrm{~s}$. We can therefore give a rough estimate of average wave speed across the array as $400 \mathrm{~m} / 1.02 \mathrm{~s} \simeq 400 \mathrm{~m} / \mathrm{s}$.

Continuing with the coherence matrices at $20 \mathrm{~Hz}$ a localized array graph $G\left(c_{\alpha=0.01}\right)$ is defined and all connected components are identified. Figure $11 \mathrm{~A}$ shows the coherent groups found over four consecutive $10.2 \mathrm{~s}$ analysis windows starting on March 11th, 10:48:48h. For clarity, only clusters with more than nine vertices are shown in order to focus on larger phenomena. The period contains a $40 \mathrm{~s}$ stretch during which a seismic vibrotruck is known to have been operating in the Southeast of the array, which is confirmed by a cluster in that 

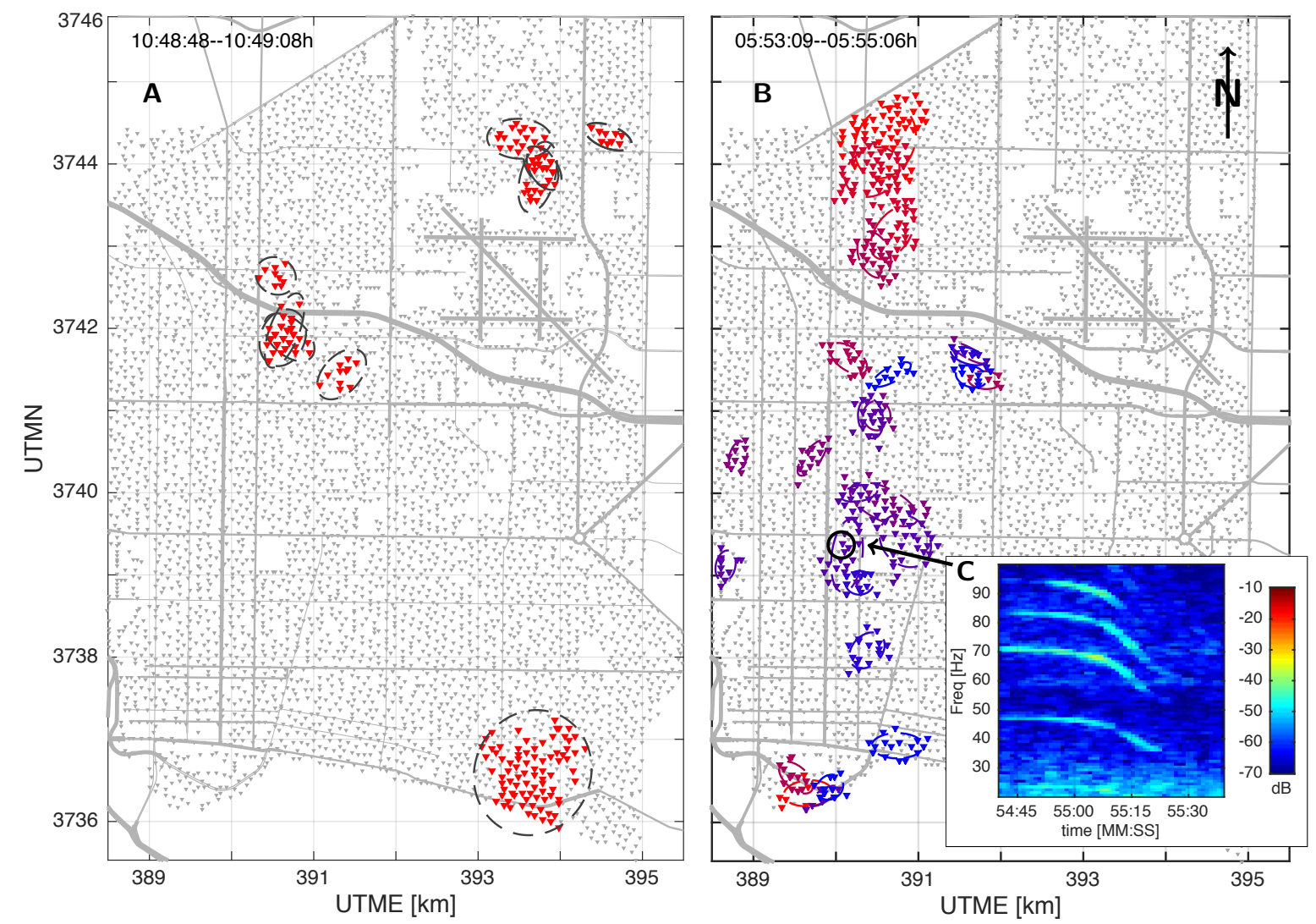

Figure 11: Connected components of the array graph are used to find coherent sensor clusters in the Long Beach geophone array. (A) The clusters at $20 \mathrm{~Hz}$ from four $10.2 \mathrm{~s}$ windows after 10:48h on March 11th. The spatial extent of the clusters $\Omega(0.5)$ is indicated by dashed ellipses (12). $(B)$ A North-to-South helicopter transect is captured in a sequence of coherent clusters at $47 \mathrm{~Hz}$ over consecutive analysis time periods (starting at 05.53h). The colors change from red to blue as the analysis windows advance in time. The arrow points to the receiver from which the spectrogram (C) was computed around the time of increased coherence. Coordinates are given in the Universal Transverse Mercator (UTM) coordinate system.

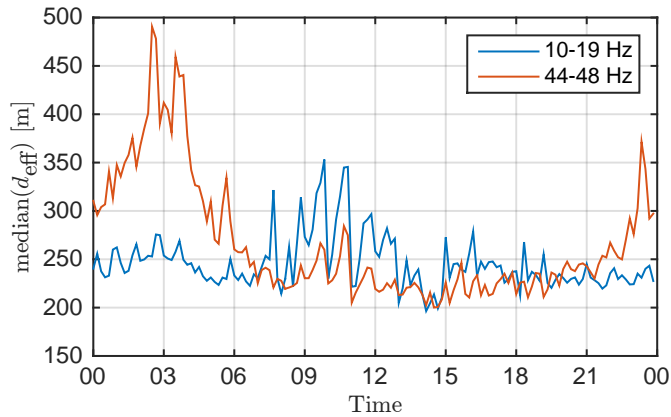

Figure 12: The median effective diameter $d_{\text {eff }}$ of detected clusters versus time-of-day (Thursday, March 10th). area. This source dominated over the background and was also broad-band (about $10-80 \mathrm{~Hz}$ ) and therefore corresponds to the type of source that was also detected with the energy-only approach reported in [18].

Figure 11B shows a sequence of coherent groups at $47 \mathrm{~Hz}$ for consecutive windows starting March 11th at $05.53 \mathrm{~h}$. They show a north-south transect over $6 \mathrm{~km}$ during the course of about $95 \mathrm{~s}$. The average velocity along the trajectory is $60 \mathrm{~m} / \mathrm{s}(134 \mathrm{mph})$. Figure $11 \mathrm{C}$ shows a spectrogram from a receiver within the trajectory of the moving source computed around the time the coherence was observed. The observed Doppler shifts of $f_{\text {high }} / f_{\text {low }} \simeq 1.4 \simeq\left(1+\frac{60}{340}\right) /\left(1-\frac{60}{340}\right)$ are consistent with the approximate velocity estimate. The narrowband harmonics at multiples of $12 \mathrm{~Hz}$ suggest that the passage of a helicopter was captured.

We perform the above analysis with the same parameterization for all frequency bins from $9.8-48.8 \mathrm{~Hz}$ for 24 hours starting at $00.00 \mathrm{~h}$ on March 10th. For every 

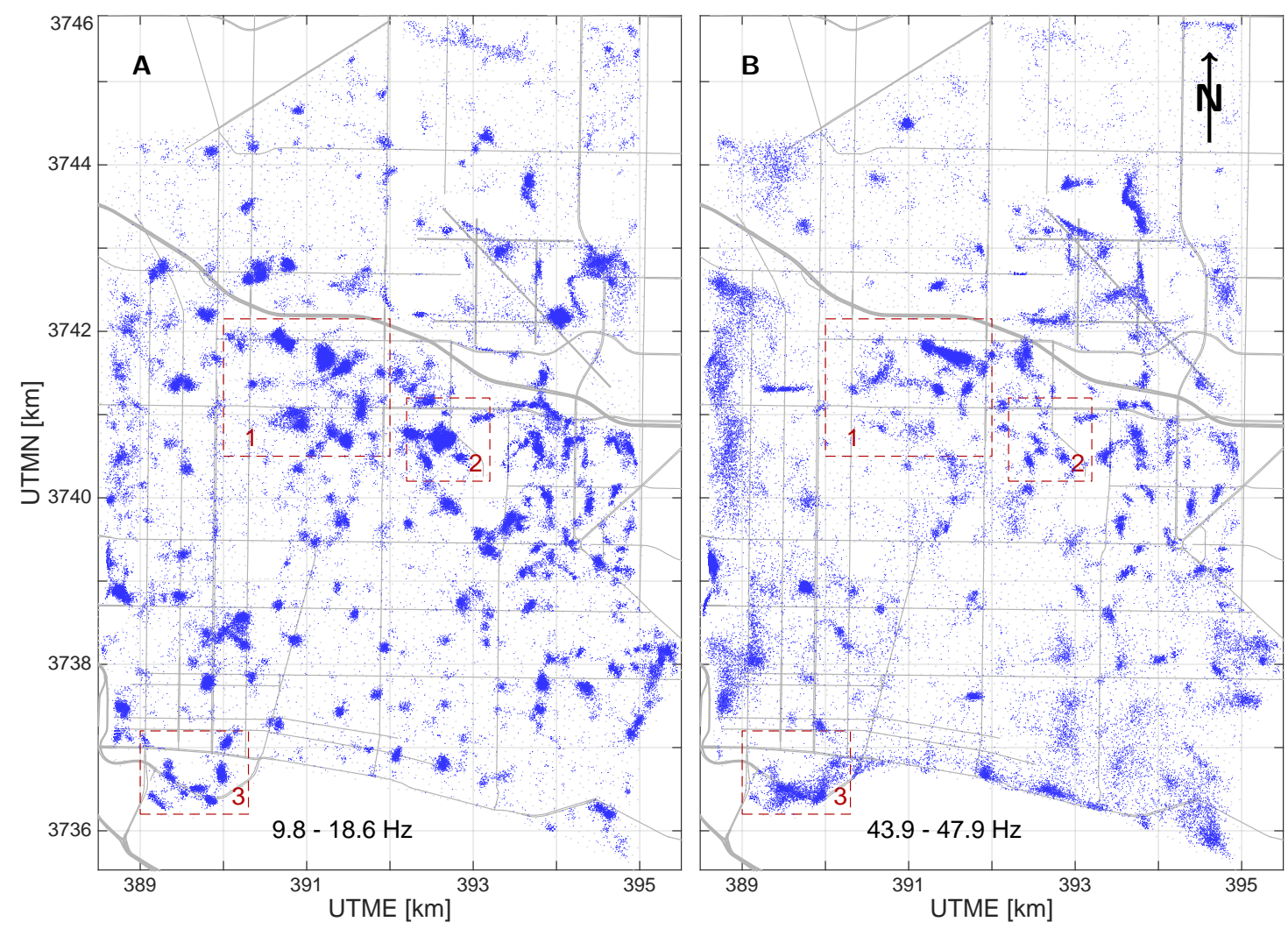

Figure 13: All clusters identified during March 10th in the frequency range 9.8-18.6 Hz $(A)$ and $43.9-47.9 \mathrm{~Hz}(B)$. Each dot represents the center of a sensor cluster. Of those clusters $90 \%$ consist of less than 19 sensors, corresponding to $0.4 \%$ of array sensors.

detected coherent sensor cluster we store the mean of the coordinates (11), the area $\Omega(0.5)$ of the cluster ellipse, and the frequency and time at which the cluster is observed. More than 150,000 clusters satisfied the conditions given in Section 2.4 (more than four vertices and more than five edges) for the analyzed time period and frequency bands. Only a small fraction these are false detections and they should be scattered randomly on the map.

Figure 12 shows the median diameter $d_{\mathrm{eff}}$ (13) versus time of day. The diameters are typically in the range $200-300 \mathrm{~m}$ but increase significantly between midnight and 5am for the higher frequency clusters (44$48 \mathrm{~Hz}$ ). We speculate that this may be due to fewer noise sources at higher frequencies during the night which would increase the SNR and hence the cluster diameter. Another possibility is that downward refracting atmospheric propagation conditions, which are more common at night, were causing signals to propagate farther at those particular frequencies.

The maps in Figure 13 show the geographic location of the identified cluster centers for the 24 hour period in the frequency bands $9.8-18.6 \mathrm{~Hz}$ and $43.9-47.9 \mathrm{~Hz}$, each containing 10 frequency bins. Three particular regions in the map are highlighted. Region 1 contains several pump jacks and drill rigs while region 2 contains a central pump facility. Note how the spatial distribution of detections differs for the two bands. Region 3 highlights the area around the Long Beach convention center. The localizations there are much less scattered for the low-frequency band which may indicate that source in those frequencies are more stationary.

\section{Conclusion}

We have proposed a model-free technique that can approximately localize weak sources within dense arrays. The approach requires that source signals exhibit significant coherence only over a small distance within the array. The support of the array coherence matrix is reinterpreted as a connectivity matrix of a graph with sensors as vertices.

For the long observation times we show that nonoverlapping clusters in this graph indicate sensor groups 
that are affected by a common source. The area spanned by these clusters can therefore serve as a location estimate. The support of the covariance matrix is estimated from short-time coherence estimates combined with an nearest neighbor approach. The latter prevents large clusters in the graph from forming by chance.

The method was verified on simulated data from a dense array with $2.8 \mathrm{~km} \times 2.8 \mathrm{~km}$ aperture and sensor spacing of $100 \mathrm{~m}$ containing four simultaneously emitting sources. For signals with SNR $=2$ at $100 \mathrm{~m}$ from the source the retrieved localization areas have a diameter of about 2-3 times the sensor separation (200-300 m). For higher SNR this diameter increases which limits resolution as the clusters of nearby sources tend to coalesce. Applied on a real data example from a $7 \mathrm{~km} \times 10 \mathrm{~km}$ seismic array with $100 \mathrm{~m}$ sensor separation, the method reveals anthropogenic source details such as a helicopter transect and oil production facilities.

\section{Acknowledgments}

The authors thank NodalSeismic LLC and Signal Hill Petroleum Inc. for sharing the data, in particular the efforts of Dan Hollis and Robert Clayton. This work is supported by NSF grant no. EAR-0944109 and by the Swiss National Science Foundation.

\section{Appendix A. Connected components and the con- nectivity matrix}

Let $G$ be a graph with $N$ vertices and let $U^{k} \in G, k=$ $1, \ldots, K$ be sub-graphs that do not overlap, $U^{k} \cap U^{l}=$ $\emptyset, \forall k \neq l$. Define the indicator vector of the vertices in $U^{k}$ as (an italic index $i$ denotes the $i$-th vertex of $G$ ):

$$
\mathbf{u}^{k}=\left[u_{1}^{k}, \ldots, u_{N}^{k}\right]^{T} \text { where } u_{i}^{k}= \begin{cases}1 & \text { if } i \in U^{k} \\ 0 & \text { if } i \notin U^{k}\end{cases}
$$

The following statements are equivalent:

1. The graph $G$ has $K$ (and only $K$ ) connected components $U^{k}$ that are not connected with each other, i.e. $e_{i j}=1$ only if $i, j \in U^{k}$.

2. $\mathbf{E}$ has a support that is equal or smaller than $\sum_{k=1}^{K} \mathbf{u}^{k} \mathbf{u}^{k^{T}}$.

Sketch of proof: Consider first the fully connected case, i.e. $u_{i}^{k}=1 \forall i \in U^{k}$. If the first statement is true, then $u_{i}^{k} u_{j}^{k}=1$ if $i, j \in U^{k}$ or 0 otherwise. But this means that $E_{i j}=\sum_{k=1}^{K} u_{i}^{k} u_{j}^{k}>0$ only if $i, j \in U^{k}$ for some $k$ and the second item is true. The other direction follows a similar logic. Because the fully connected component contains all possible edges within a set of vertices any lesser connected components will contain a subset of those edges, i.e. the connectivity matrix can only have fewer non-zero values.

\section{Appendix B. Algebra on indicator vectors}

Define the support indicator function of a vector $\mathbf{v} \in$ $\mathbb{C}^{N}$ :

$$
\hat{\mathbf{v}}=\mathcal{I}(\mathbf{v}) \text { where } \hat{\mathbf{v}}_{i}= \begin{cases}1 & \text { if } \mathbf{v}_{i} \neq 0 \\ 0 & \text { if } \mathbf{v}_{i}=0\end{cases}
$$

and analogously an indicator function $\mathcal{I}(\mathbf{V})$ for a matrix V. We then have:

$$
\begin{aligned}
\mathcal{I}\left(\mathbf{v} \mathbf{w}^{H}\right) & =\hat{\mathbf{v}} \hat{\mathbf{w}}^{T} \\
\mathcal{I}(a \mathbf{v}) & =\hat{\mathbf{v}} \quad(\text { for } a \neq 0) \\
\mathcal{I}(\mathbf{V}+\mathbf{W}) & =\hat{\mathbf{V}}+\hat{\mathbf{W}}-(\hat{\mathbf{V}} \circ \hat{\mathbf{W}}),
\end{aligned}
$$

where $\circ$ is the Hadamard operator (element-wise multiplication) and assuming we don't have $V_{i j}=-W_{i j} \neq 0$. Assume that the supports of $\mathbf{v}$ and $\mathbf{w}$ do not overlap, i.e. $\hat{\mathbf{v}} \circ \hat{\mathbf{w}}=\mathbf{0}$, the zero-vector. Therefore

$$
\left(\hat{\mathbf{v}} \hat{\mathbf{v}}^{T}\right) \circ\left(\hat{\mathbf{W}} \hat{\mathbf{W}}^{T}\right)=(\hat{\mathbf{v}} \circ \hat{\mathbf{w}})(\hat{\mathbf{v}} \circ \hat{\mathbf{W}})^{T}=\mathbf{0}
$$

is the zero matrix and consequently, from (B.4) and (B.5)

$$
\begin{aligned}
\mathcal{I}\left(\mathbf{v} \mathbf{v}^{H}+\mathbf{w} \mathbf{w}^{H}\right) & =\hat{\mathbf{v}} \hat{\mathbf{v}}^{T}+\hat{\mathbf{w}} \hat{\mathbf{w}}^{T}-\left(\hat{\mathbf{v}} \hat{\mathbf{v}}^{T} \circ \hat{\mathbf{w}} \hat{\mathbf{w}}^{T}\right) \\
& =\hat{\mathbf{v}} \hat{\mathbf{v}}^{T}+\hat{\mathbf{w}} \hat{\mathbf{w}}^{T} .
\end{aligned}
$$

In other words, if the support of vectors $\mathbf{v}$ and $\mathbf{w}$ do not overlap, then the support of the sum of their outer products is simply the sum of the outer products of their support-indicators.

\section{References}

[1] R. A. Kerr, Geophysical exploration linking deep earth and backyard geology, Science 340 (6138) (2013) 1283-1285.

[2] H. P. Bucker, Use of calculated sound fields and matched-field detection to locate sound sources in shallow water, The Journal of the Acoustical Society of America 59 (2) (1976) 368-373.

[3] A. Baggeroer, W. Kuperman, P. Mikhalevsky, An overview of matched-field methods in ocean acoustics, IEEE Journal of Oceanic Engineering 18 (4) (1993) 401-424. doi: $10.1109 / 48.262292$.

[4] J. C. Chen, K. Yao, R. E. Hudson, Acoustic source localization and beamforming: Theory and practice, EURASIP Journal on Advances in Signal Processing 2003 (4) (2003) 1-12.

[5] A. Beck, P. Stoica, J. Li, Exact and approximate solutions of source localization problems, Signal Processing, IEEE Transactions on 56 (5) (2008) 1770-1778. 
[6] J. F. Claerbout, Toward a unified theory of reflector mapping, Geophysics 36 (3) (1971) 467-481.

[7] G. McMechan, Migration by extrapolation of time-dependent boundary values, Geophysical Prospecting 31 (3) (1983) 413420.

[8] P. M. Shearer, Global seismic event detection using a matched filter on long-period seismograms, Journal of Geophysical Research: Solid Earth 99 (B7) (1994) 13713-13725.

[9] D. B. Harris, T. Kvaerna, Superresolution with seismic arrays using empirical matched field processing, Geophysical Journal International 182 (3) (2010) 1455-1477.

[10] M. Corciulo, P. Roux, M. Campillo, D. Dubucq, W. A. Kuperman, Multiscale matched-field processing for noise-source localization in exploration geophysics, Geophysics 77 (5) (2012) KS33-KS41. doi:10.1190/geo2011-0438.1.

[11] M. A. H. Hedlin, D. Drob, K. Walker, C. de Groot-Hedlin, A study of acoustic propagation from a large bolide in the atmosphere with a dense seismic network, Journal of Geophysical Research: Solid Earth 115 (B11).

[12] K. T. Walker, R. Shelby, M. A. H. Hedlin, C. de Groot-Hedlin, F. Vernon, Western U.S. infrasonic catalog: Illuminating infrasonic hot spots with the USArray, Journal of Geophysical Research: Solid Earth 116 (B12).

[13] D. F. Gingras, P. Gerstoft, N. L. Gerr, Electromagnetic matchedfield processing: Basic concepts and tropospheric simulations, IEEE Transactions on Antennas and Propagation 45 (10) (1997) 1536-1545.

[14] P. Valtr, P. Pechac, V. Kvicera, M. Grabner, Estimation of the refractivity structure of the lower troposphere from measurements on a terrestrial multiple-receiver radio link, IEEE Transactions on Antennas and Propagation 59 (5) (2011) 1707-1715.

[15] J. Capon, High-resolution frequency-wavenumber spectrum analysis, Proc. IEEE 57 (8) (1969) 1408-1418.

[16] R. O. Schmidt, Multiple emitter location and signal parameter estimation, IEEE Trans. Antennas Propag. 34 (3) (1986) 276280. doi:10.1109/tap.1986.1143830.

[17] D. Li, Y. H. Hu, Energy-based collaborative source localization using acoustic microsensor array, EURASIP Journal on Advances in Signal Processing 2003 (4) (2003) 985029. doi:10.1155/S1110865703212075.

[18] N. Riahi, P. Gerstoft, The seismic traffic footprint: Tracking trains, aircraft, and cars seismically, Geophys. Res. Lett. 42 (8) (2015) 2674-2681. doi:10.1002/2015gl063558.

[19] D. I. Shuman, S. K. Narang, P. Frossard, A. Ortega, P. Vandergheynst, The emerging field of signal processing on graphs: Extending high-dimensional data analysis to networks and other irregular domains, IEEE Signal Processing Magazine 30 (3) (2013) 83-98.

[20] A. Sandryhaila, J. M. Moura, Big data analysis with signal processing on graphs: Representation and processing of massive data sets with irregular structure, IEEE Signal Processing Magazine 31 (5) (2014) 80-90.

[21] U. von Luxburg, A tutorial on spectral clustering, Statistics and Computing 17 (4) (2007) 395-416. doi:10.1007/s11222-0079033-z.

[22] W. H. Press, Numerical recipes : the art of scientific computing, 3rd Edition, Cambridge University Press, Cambridge, UK ; New York, 2007.

[23] A. D. Chave, D. J. Thomson, M. E. Ander, On the robust estimation of power spectra, coherences, and transfer functions, Journal of Geophysical Research: Solid Earth 92 (B1) (1987) 633-648.

[24] M. E. Newman, The structure and function of complex networks, SIAM review 45 (2) (2003) 167-256.

[25] S. E. Schaeffer, Graph clustering, Computer Science Review
1 (1) (2007) $27-64$.

[26] D. Friedlander, C. Griffin, N. Jacobson, S. Phoha, R. R. Brooks, Dynamic agent classification and tracking using an ad hoc mobile acoustic sensor network, EURASIP Journal on Advances in Signal Processing 2003 (4) (2003) 1-7.

[27] M. E. J. Newman, Finding community structure in networks using the eigenvectors of matrices, Phys. Rev. E 74 (2006) 036104. doi:10.1103/PhysRevE.74.036104.

[28] S. Fortunato, Community detection in graphs, Physics Reports $486(3-5)(2010) 75-174$.

[29] S. Sobolevsky, R. Campari, A. Belyi, C. Ratti, General optimization technique for high-quality community detection in complex networks, Phys. Rev. E 90 (2014) 012811.

[30] F.-C. Lin, V. C. Tsai, B. Schmandt, Z. Duputel, Z. Zhan, Extracting seismic core phases with array interferometry, Geophysical Research Letters 40 (6) (2013) 1049-1053. doi:10.1002/grl.50237.

[31] B. Schmandt, R. W. Clayton, Analysis of teleseismic P waves with a 5200-station array in Long Beach, California: Evidence for an abrupt boundary to Inner Borderland rifting, J. of Geophys. Res.: Solid Earth 118 (10) (2013) 5320-5338.

[32] D. Bowden, V. Tsai, F. Lin, Site amplification, attenuation, and scattering from noise correlation amplitudes across a dense array in long beach, ca, Geophysical Research Letters 42 (5) (2015) 1360-1367.

[33] N. Nakata, J. P. Chang, J. F. Lawrence, P. Boué, Body wave extraction and tomography at Long Beach, California, with ambient-noise interferometry, Journal of Geophysical Research: Solid Earth 120 (2) (2015) 1159-1173.

[34] J. P. Chang, S. A. L. de Ridder, B. L. Biondi, High-frequency Rayleigh-wave tomography using traffic noise from Long Beach, California, Geophysics 81 (2) (2016) B43-B53. 